\title{
MicroRNA-29c targets $\beta$-site amyloid precursor protein-cleaving enzyme 1 and has a neuroprotective role in vitro and in vivo
}

\author{
GUOSHUAI YANG ${ }^{1,2}$, YANMIN SONG ${ }^{3}$, XIAOYAN ZHOU ${ }^{2}$, YIDONG DENG $^{1}$, \\ TAO LIU ${ }^{1}$, GUOHU WENG ${ }^{1}$, DAN YU ${ }^{2}$ and SUYUE PAN ${ }^{1}$ \\ ${ }^{1}$ Department of Neurology, Nanfang Hospital, Southern Medical University, Guangzhou, Guangdong 510515; \\ ${ }^{2}$ Department of Neurology, Haikou People's Hospital, Haikou, Hainan 570208; ${ }^{3}$ Department of Neurology, \\ Xiangya Hospital, Central South University, Changsha, Hunan 410008, P.R. China
}

Received July 14, 2014; Accepted March 24, 2015

DOI: $10.3892 / \mathrm{mmr} .2015 .3728$

\begin{abstract}
Alzheimer's disease (AD), characterized by $\beta$-amyloid deposition and neurodegeneration, is the most common cause of dementia worldwide. Emerging evidence suggests that ectopic expression of micro (mi)RNAs is involved in the pathogenesis of AD. There is increasing evidence that miRNAs expressed in the brain are involved in neuronal development, survival and apoptosis. The expression of $\beta$-site amyloid precursor protein-cleaving enzyme 1 (BACE1) is regulated by dysregulated miRNAs in the brain. The present study determined the expression levels of the miRNA-29 (miR-29) family in peripheral blood samples of patients with $\mathrm{AD}$ and demonstrated a marked decrease in the expression of miR-29c compared with age-matched controls. In addition, a significant increase in the exprssion of BACE1 was observed in the peripheral blood of patients with AD. Correlation analysis revealed that the expression of $\mathrm{miR}-29 \mathrm{c}$ was negatively correlated with the protein expression of BACE1 in the peripheral blood samples from patients with AD. The present study also investigated the role of miR-29 on hippocampal neurons in vitro and in vivo. The results demonstrated that the upregulation of miR-29c promoted learning and memory behaviors in SAMP8 mice, at least partially, by increasing the activity of protein kinase A/cAMP response element-binding protein, involved in neuroprotection. This evidence suggested that miR-29c may be a promising potential therapeutic target against AD.
\end{abstract}

\section{Introduction}

Alzheimer's disease (AD) is the most common cause of dementia worldwide and is characterized by pathological

Correspondence to: Professor Suyue Pan, Department of Neurology, Nanfang Hospital, Southern Medical University, 1838 Guangzhou Avenue, Guangzhou, Guangdong 510515, P.R. China

E-mail: doctorpansuyue@163.com; southpansuyue@163.com

Key words: Alzheimer's disease, microRNA, $\beta$-site amyloid precursor protein-cleaving enzyme 1 , memory, protein kinase $\mathrm{A}$ $\beta$-amyloid (A $\beta$ ) deposition and neurodegeneration (1). Although the precise mechanisms underlying neurodegeneration in $\mathrm{AD}$ remain to be elucidated, the role of the complex interaction between genetic and environmental factors has been supported in previous studies (2-4). In addition, emerging evidence has indicated the critical involvement of the ectopic expression of miRNA in the pathogenesis of AD (5).

MicroRNAs (miRNAs), a class of endogenous, small ( $22 \mathrm{nt})$, noncoding RNAs, mediate post-transcriptional regulation of protein-coding genes by binding to the $3^{\prime}$ untranslated region ( $3^{\prime} \mathrm{UTR}$ ) of target genes. This leads to translational inhibition or mRNA degradation, reducing the production of hundreds of proteins (6). There is evidence indicating that miRNAs expressed in the brain are involved in neuronal development, survival and apoptosis (7). $\beta$-site amyloid precursor protein-cleaving enzyme1 (BACE1), a rate-limiting enzyme involved in the production of $\mathrm{A} \beta$, is important in the pathogenesis of AD (8). Several downregulated miRNAs have been demonstrated in the brain, including the miR-107 (9) and miR-29 families (5), which regulate the expression of BACE1 and consequently affect the production of $A \beta$. Of the presently identified miRNAs, $\sim 70 \%$ are expressed in the brain, however, the pathological implications of dysregulated miRNA expression in AD remain to be fully elucidated (10).

The present study aimed to investigate the neuroprotective role of miR-29c in AD. The results of the present study may be therapeutically beneficial for the treatment of AD.

\section{Materials and methods}

Blood samples. A total of 60 samples of peripheral blood were collected from the median cubital veins of patients with AD and from normal age-matched individuals ( $>65$ years old) Haikou People's Hospital (Hainan, China). The patients with AD were aged between 65 and 86 years (male, $n=17$; female $\mathrm{n}=13$ ), and the normal control individuals were aged between 65 and 74 years (male, $n=19$; female, $n=11$ ). Individuals with significant illness, including diabetes, heart disease, stroke or cancer, were excluded from the present study. All the samples were collected according to the legislation and ethical boards of Haikou People's Hospital. Written informed consent was 
obtained from the patients. The samples were stored at $-80^{\circ} \mathrm{C}$ until use.

Cell culture and treatment. Primary hippocampal neurons were obtained from the hippocampi of SAMR1 mice at embryonic day 15 . Briefly, the hippocampi were mechanically dissociated and treated with $0.2 \%$ trypsin (Sigma-Aldrich, St. Louis, MO, USA) for $15 \mathrm{~min}$ at $37^{\circ} \mathrm{C}$ in phosphate-buffered saline (PBS). The hippocampal cells were collected by centrifugation at $118 \times \mathrm{g}$ for $5 \mathrm{~min}$, and then $2 \times 10^{5}$ cells $/ \mathrm{ml}$ were washed in Dulbecco's modified Eagle's medium (DMEM; Invitrogen Life Technologies, Carlsbad, CA, USA), containing $10 \%$ fetal bovine serum (FBS; Invitrogen Life Technologies), and resuspended in DMEM supplemented with 10\% FBS and $100 \mathrm{kU} / 1$ penicillin and streptomycin (Sigma-Aldrich). The cells were cultured in a humidified atmosphere of $95 \%$ air and $5 \% \mathrm{CO}_{2}$. Ectopic expression of miR-29c in cells was introduced by transfection with miR-29c mimics or inhibitors using Lipofectamine 2000 (Invitrogen Life Technologies).

Animals and treatment. The SAMR1 and SAMP8 mice were obtained from the Animal Center of Beijing University Medical Department (Beijing, China) and housed in standard conditions (12 h light/dark; $25 \pm 1^{\circ} \mathrm{C} ; 50 \%$ humidity). Male 8-month-old SAMP8 $(n=6)$ and SAMR1 $(n=6)$ mice were used. The anesthetized $(40 \mathrm{mg} / \mathrm{kg} 1 \%$ pentobarbital sodium; Sigma-Aldrich) SAMP8 mice were positioned in a stereotaxic apparatus (model 68016; RWD Life Science, Shenzhen, China), and $1.5 \mu \mathrm{l}$ PBS, containing either $0.5 \mathrm{nmol}$ miR-29c mimic or a scrambled control (Intvitrogen Life Technologies, Carlsbad, CA, USA), was injected for $8 \mathrm{~min}$ into the third ventricle. The control SAMR1 mice received an equal volume of the vehicle (PBS only). The present study was approved by the ethics committee of Southern Medical University (Guangzhou, China), in compliance with the National Institute of Health Guide for the Care and Use of Laboratory Animals (11).

Reverse transcription-quantitative polymerase chain reaction (RT-qPCR). The total RNA was extracted from the indicated tissues or cells using RNA Extraction reagent, according to the manufacturer's instructions (CWBio, Beijing, China). The mRNA expression of BACE1 was detected using a SYBR green qPCR assay (CWBio). RevertAid First Strand cDNA Synthesis kit (Thermo Fisher Scientific, Waltham, MA, USA) was used to reverse transcribe the RNA. The expression of $\beta$-actin was used as an endogenous control. The specific primers used were as follows: BACE1, forward 5'-A ATTCGAAATGGCCCAAGCCCTGCCCT-3' and reverse 5'-AGGGATCCGGGCCTCCTCACTTCAGCAG-3', and $\beta$-actin, forward 5'-CATTAAGGAGAAGCTGTGCT-3' and reverse 5'-GTTGAAGGTAGTTTCGTGGA-3'. A MiScript SYBR-Green PCR kit (Ribobio, Co., Ltd., Guangzhou, China) was used for qPCR to detect the expression levels of miR-29c. The specific primer sets for miRNA-29c and U6 were purchased from GeneCopoeia (Rockville, MD, USA). The expression of U6 was used as an endogenous control. A total of $2 \mu \mathrm{lDNA}(50 \mathrm{ng} / \mu \mathrm{l})$ was used to analyze the expression levels on a CFX96 Real-Time system (Bio-Rad Laboratories, Inc., Hercules, CA, USA). The PCR conditions were as follows: $95^{\circ} \mathrm{C}$ for $5 \mathrm{~min}$, followed by 40 cycles at $95^{\circ} \mathrm{C}$ for $30 \mathrm{sec}, 58^{\circ} \mathrm{C}$ for $30 \mathrm{sec}$ and $72^{\circ} \mathrm{C}$ for $30 \mathrm{sec}$, and finally $72^{\circ} \mathrm{C}$ for $10 \mathrm{~min}$. The data were then analyzed using the $2^{-\Delta \Delta \mathrm{CT}}$ method (12).

ELISA determination of $A \beta$. Mouse $\mathrm{A} \beta$ immunoassay kits (Invitrogen Life Technologies) were used to determine the levels of $A \beta$ in the primary cultured hippocampal cells and the brain tissues of the mice, according to the manufacturer's instructions. Briefly, the media in the cultured cells or the supernatants of the indicated tissues were used to measure the total protein quantities of each sample. The samples and the $\mathrm{A} \beta$ antibody were incubated overnight at $4^{\circ} \mathrm{C}$, prior to incubation with horseradish peroxidase (HRP)-labeled anti-rabbit antibody for $30 \mathrm{~min}$ at room temperature. The wells were then developed using $100 \mu \mathrm{l}$ tetramethylbenzidine reagent in the dark, and the absorbance was measured at $450 \mathrm{~nm}$ (Synergy $^{\mathrm{TM}}$ Mx; BioTek Instrument, Inc., Winooski, VT, USA).

Western blotting. The total protein was extracted from the indicated cells or tissues using cold radioimmunoprecipitation lysis buffer (CWBio). A bicinchoninic acid protein assay kit (Pierce Biotechnology, Rockford, IL, USA) was used to determine the protein concentrations. The protein $(60 \mu \mathrm{g})$ was subsequently separated on a $10 \%$ SDS-PAGE gel (Wuhan Boster Biological Technology, Ltd., Wuhan, China) and transferred onto a nitrocellulose membrane (Wuhan Boster Biological Technology, Ltd.). The membrane was blocked in 5\% nonfat dried milk in PBS for $4 \mathrm{~h}$, and was incubated with the following primary antibodies overnight at $4^{\circ} \mathrm{C}$ : Rabbit anti-protein kinase $\mathrm{A}$ (PKA; 1:1,00; cat. no. 4781; Cell Signaling Technology, Inc., Danvers, MA, USA), rabbit anti-BACE (1:500; cat. no. 5606; Cell Signaling Technology, Inc.), rabbit anti-cAMP response element binding protein (CREB; 1:500; cat. no. 4820; Cell Signaling Technology, Inc.), rabbit anti-phosphorlyated (p) CREB (1:500; cat.no.9198; Cell Signaling Technology,Inc.) and mouse anti- $\beta$-actin (1:3,000; cat. no. BM0627; Wuhan Boster Biological Technology, Ltd.). The membranes were washed with Tris-buffered saline containing $0.1 \%$ Tween and incubated with HRP-labeled goat anti-rabbit (cat. no. A12004-1; 1:2,000) and goat anti-mouse (cat. no. A12003-1; 1:3,000) secondary antibodies (Epigentek, Farmingdale, NY, USA) for $2 \mathrm{~h}$ at room temperature. Enhanced chemiluminescence reagent (Wuhan Boster Biological Technology, Ltd.) was used to detect the signal on the membrane. The expression data were analyzed by densitometry using Image-Pro plus software 6.0 (Media Cybernetics, Inc., Rockville, MD, USA) and normalized against that of the internal control.

Dual luciferase reporter assay. Wild-type (wt) and mutant (mut) 3'-UTRs of BACE1 were constructed, which were inserted into the dual luciferase reporter vector (Promega Corporation, Madison, WI, USA). Briefly, to generate the wt-BACE1-3'-UTR, the 3'-UTR was amplified and cloned into the XbaI (Thermo Fisher Scientific) site of the pGL3-control vector (Promega Corporation), downstream of the luciferase gene. The mut-BACE1-3'-UTR was generated from wt-BACE1-3'-UTR by site-directed mutagenesis, by Genecopeoia (Guangzhou, China). For the luciferase assay, 100,000 cells were cultured to $\sim 70 \%$ confluence in 24 -well plates. Following culture, the cells were co-transfected with the miR-29c mimic and either the wt or mut 3'-UTR of the BACE1 
dual luciferase reporter vector. Following incubation for $5 \mathrm{~h}$ with the transfection reagent/DNA complex, the medium was replaced with fresh medium, containing 10\% FBS. At $48 \mathrm{~h}$ post-transfection, a Dual Luciferase Reporter Gene Assay kit (BioVision, Milpitas, CA, USA) was used to determine the luciferase activities in each group on a luminometer (Elecsys 2010; Roche Diagnostics, Basel, Switzerland). The activity of Renilla luciferase was normalized against that of firefly luciferase.

Cell proliferation assay. For all the groups, 5,000-6,000 cells/well were seeded into a 96-well plate. Following treatment, the plates were incubated for $0,12,24$, 48 or $72 \mathrm{~h}$ at $37^{\circ} \mathrm{C}$ with $5 \% \mathrm{CO}_{2}$. To assess cell proliferation, an MTT assay was performed, according to the manufacturer's instructions. MTT reagent $(20 \mu \mathrm{l} ; 5 \mathrm{mg} / \mathrm{ml}$; Wuhan Boster Biological Technology, Ltd.) in $200 \mu \mathrm{l} \mathrm{FBS-free} \mathrm{medium}$ was added to each well and incubated for $4 \mathrm{~h}$ at $37^{\circ} \mathrm{C}$. The medium was then removed and $100 \mu$ l dimethyl sulfoxide (Wuhan Boster Biological Technology, Ltd.) was added. The absorbance was detected at $490 \mathrm{~nm}$ using a microplate reader (Elecsys 2010). The assay was repeated three times in triplicate wells.

Y-maze test. The Y-maze apparatus (model RD1102-YM-M; Mobiledatum Co., Ltd., Shanghai, China) was constructed from wood, painted in black, and had three arms at $120^{\circ}$ angles. Each arm was $50 \mathrm{~cm}$ long, $15 \mathrm{~cm}$ high, $5 \mathrm{~cm}$ wide. The mice were initially placed at the end of one arm and allowed to move freely for $10 \mathrm{~min}$. The series of arm entries was recorded using a video camera (model HDR-PJ790E; Sony, Tokyo, Japan). Spontaneous alternation was defined as: Successive entries into the three arms in overlapping triplet sets. The alternation percentages were determined as the ratios of actual alternations to maximum alternations, multiplied by 100. The percentages of alternation behaviors were recorded by an observer, in a blinded manner, followed by statistical analysis.

Statistical analysis. Statistical analyses were performed using Graphpad Prism 5 software (Graphpad Software, Inc., San Diego, CA, USA) and the data are expressed as the mean \pm standard deviation. An unpaired two-tailed Student's t-test was used to analyze the differences between the samples. $\mathrm{P}<0.05$ was considered to indicate a statistically significant difference.

\section{Results}

Levels of miR-29c are negatively correlated with protein levels of BACE1 in the blood of patients with AD. The expression levels of miR-29 were detected using SYBR green qPCR analysis. In the sample of 30 patients with AD and 30 normal individuals, no significant differences were observed in the expression levels of miR-29a and miR-29b (Fig. 1A and B), however, the results demonstrated that miR-29c was significantly decreased in 27 (90\%) of the peripheral blood samples from the patients with $\mathrm{AD}$, compared with the paired normal blood samples (Fig. 1C). However. In addition, an ELISA was used to measure the protein expression levels of BACE1. As
A

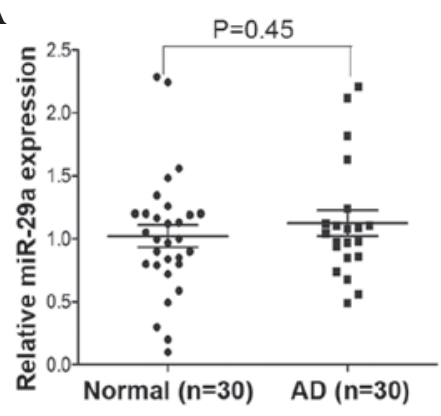

B
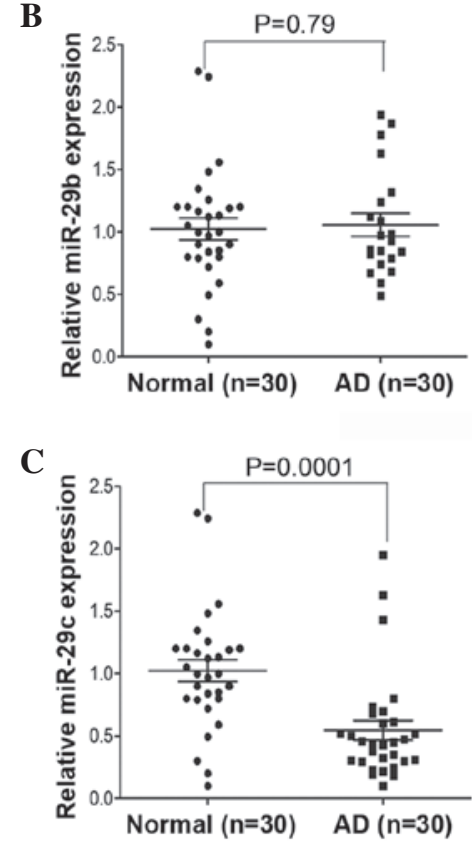

D

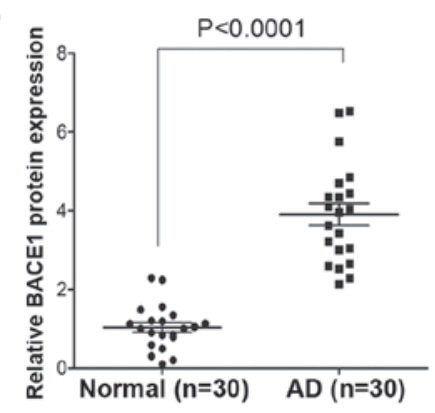

E

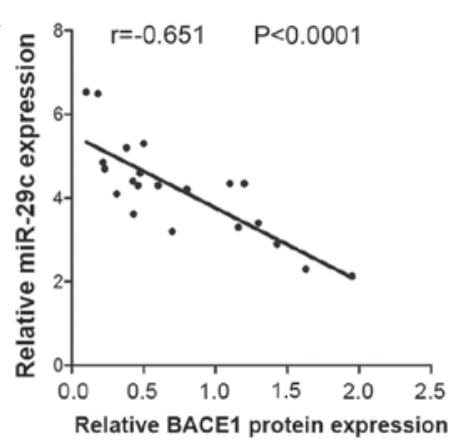

Figure 1. Correlation of miR-29c and BACE1 in patients with AD (A) Relative expression levels of miR-29a, (B) miR-29b and (C) miR-29c in blood samples. (D) Relative protein expression levels of BACE1 in blood samples. (E) Correlation analysis of the expression levels of miR-29c and BACE1. The data are expressed as the mean \pm standard deviation. BACE1, $\beta$-site amyloid precursor protein-cleaving enzyme $1 ;$ miR, microRNA; $A D$, Alzheimer's disease. 
A
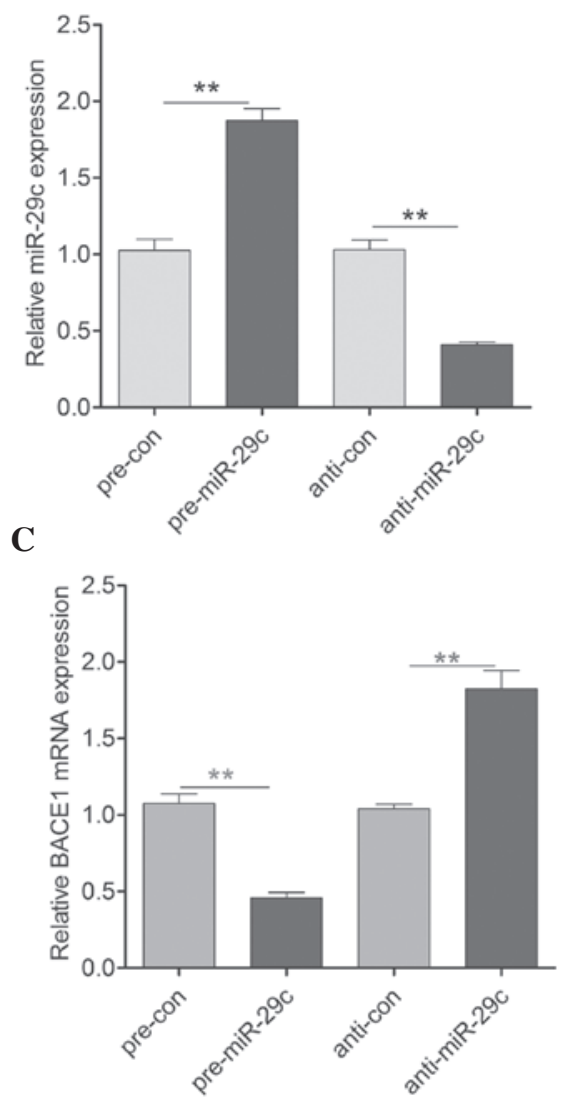

$\mathbf{E}$

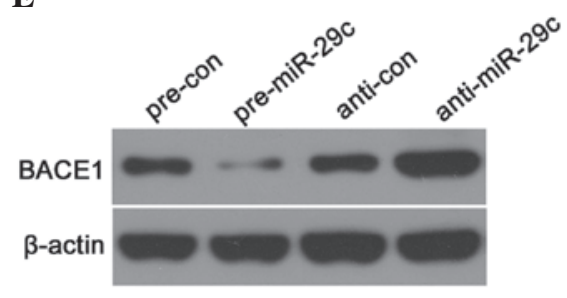

B

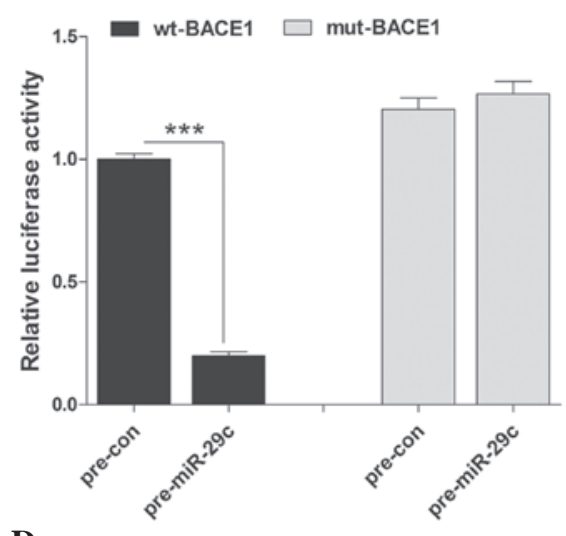

D
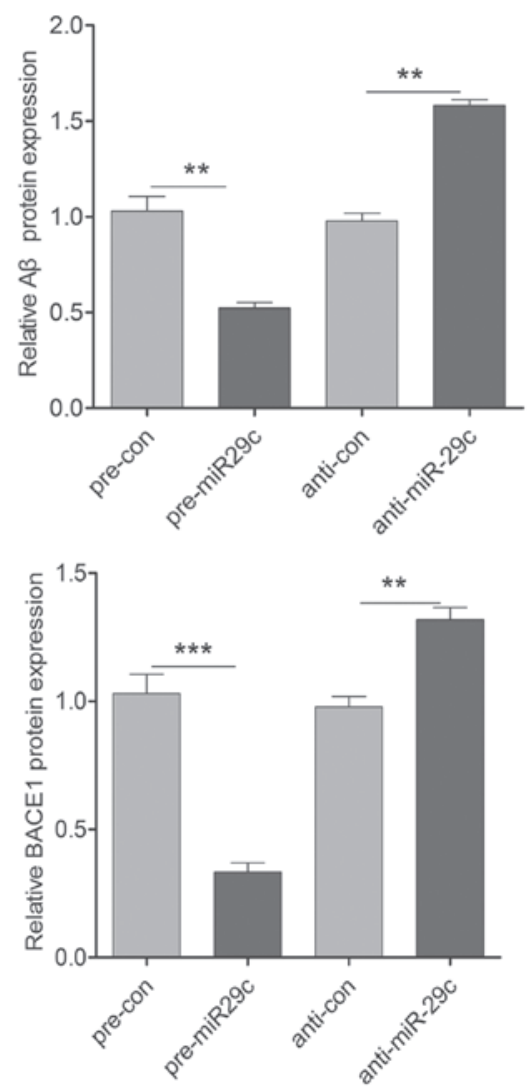

Figure 2. miR-29c directly targets the 3'UTR of BACE1. (A) RT-qPCR was performed to detect the expression levels of miR-29c following treatment with pre-miR-29c or anti-miR-29c in primary cultured hippocampal cells. (B) Repression of luciferase activity by the BACE1 3'UTR was dependent on miR-29c. Mutated BACE1 3'UTR abrogated the miR-29c mediated repression of luciferase activity. (C) RT-qPCR was performed to detect the mRNA expression levels of BACE1 following treatment with pre-miR-29c or anti-miR-29c. (D) ELISA was performed to detect the protein expression levels of $\beta$-amylase in the cultured medium following treatment with pre-miR-29c or anti-miR-29c. (E) Western blotting and quantification of the protein expression levels of BACE1 following treatment with pre-miR-29c or anti-miR-29c. The data are expressed as the mean \pm standard deviation ${ }^{* *} \mathrm{P}<0.01$ and ${ }^{* * * *} \mathrm{P}<0.001$, vs. control). BACE1, $\beta$-site amyloid precursor protein-cleaving enzyme 1; miR, microRNA; UTR, untranslated region; RT-qPCR, reverse transcription-quantitative polymerase chain reaction; con, control; wt, wild-type; mut, mutant.

shown in Fig. 1D, the protein expression levels of BACE1 were significantly increased in the peripheral blood from patients with AD, compared with the normal control individuals. In addition, the expression of miR-29c was negatively correlated with the protein expression of BACE1 in the peripheral blood of the patients with AD (Fig. 1E).

miR-29c regulates the expression of BACE1 by directly targeting its 3'UTR. To investigate whether miR-29c targets the 3'UTR of BACE1, the present study cloned the 3'UTR of BACE1 downstream to a luciferase reporter gene (wt-BACE1). The mutant version (mut-BACE1), with binding site mutagenesis, was also constructed. To determine whether miR-29c regulated the expression of BACE1 at the transcriptional or translational level, the wt-BACE1 vector and either the pre-miR-29c mimic or scramble control (anti-miR-29c)were co-transfected into primary cultured hippocampal cells. As shown in Fig. 2A, the transfection efficiency was satisfactory for further investigation. The luciferase activity of the pre-miR-29c transfected cells was 
A

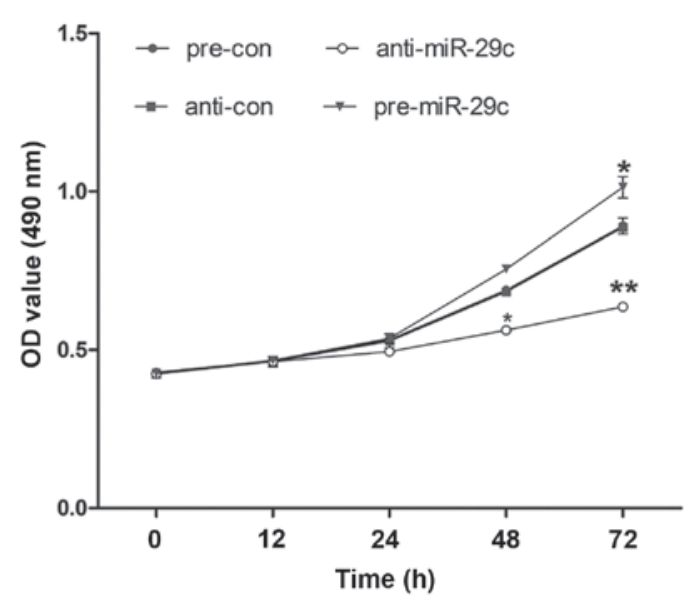

B
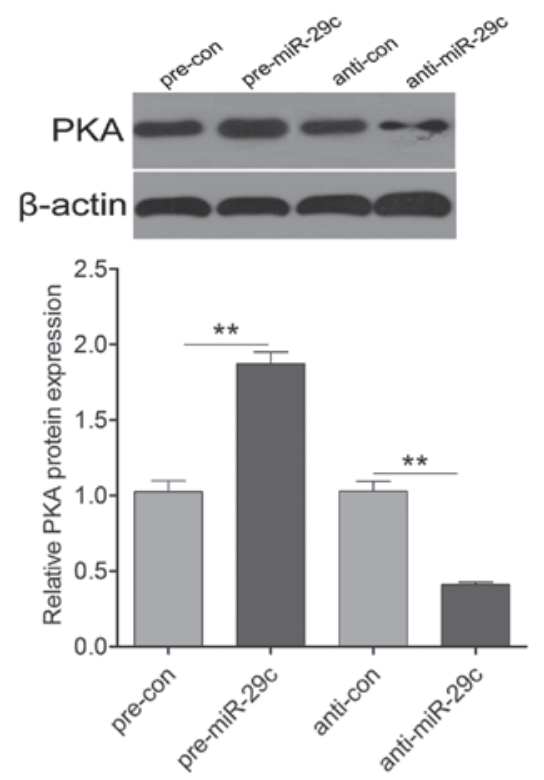

C

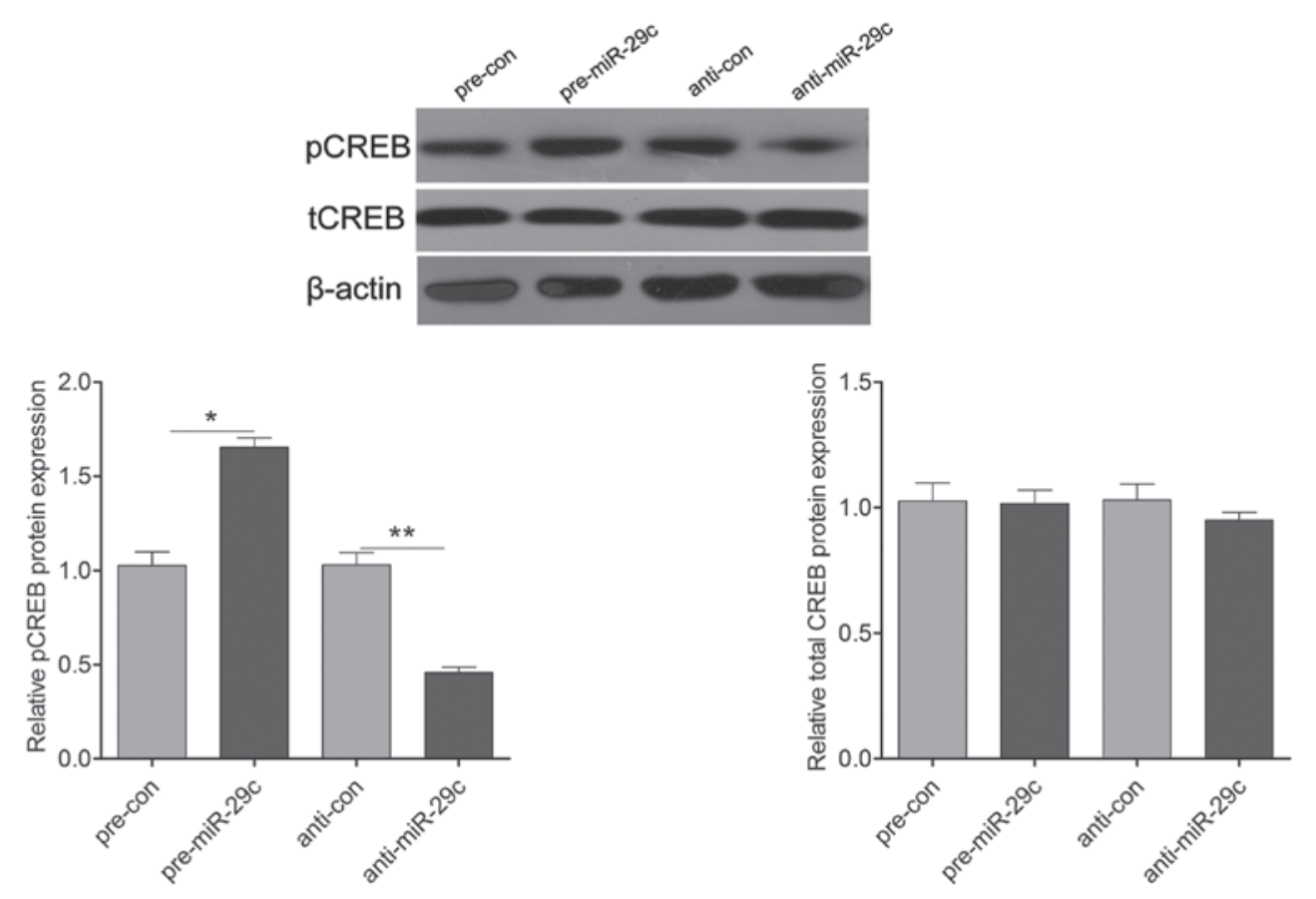

Figure 3. Ectopic expression of miR-29c regulates cell proliferation via PKA/CREB signaling. (A) An MTT assay was performed to measure cell proliferation. (B) Western blotting and quantification of the protein expression of PKA following treatment with pre-miR-29c or anti-miR-29c. (C) Western blotting and quantification of the protein expression levels of pCREB and total CREB following treatment with pre-miR-29c or anti-miR-29c. The data are expressed as the mean \pm standard deviation ( $\mathrm{P}<0.05$ and ${ }^{* *} \mathrm{P}<0.01$, vs. control). PKA, protein kinase A; CREB, cAMP response element-binding protein; $\mathrm{p}$, phosphorylated; t, total; con, control; miR, microRNA; OD, optical density.

significantly reduced, compared with the scramble control cells (Fig. 2B). Additionally, The results demonstrated that the overexpression of miR-29c significantly reduced the mRNA and protein expression levels of BACE1, whereas downregulation of miR-29c increased the mRNA and protein expression levels of BACE1 (Fig. 2C). In addition, the protein expression of $A \beta$ in the culture medium was determined. The protein expression of $A \beta$ was decreased by the upregulation of miR-29c and increased by downregulation of miR-29c (Fig. 2D). These results suggested that miR-29c regulated the expression of BACE1 (Fig.2E) at the transcriptional level by directly targeting its $3^{\prime} \mathrm{UTR}$.
miR-29c promotes cell proliferation via PKA signaling. To determine whether miR-29c regulated hippocampal neuron proliferation, an MTT assay was performed by transfecting either pre-miR-29c or anti-miR-29c into the primary cultured hippocampal cells. Pre-miR-29c transfection exhibited a significant promotion of cell proliferation, compared with the control cells, whereas transfection with anti-miR-29c led to a significant inhibition on cell proliferation (Fig. 3A). To further investigate the molecular mechanism underlying miR-29c induction on cell growth, the expression levels of molecules associated with PKA signaling were detected. As shown in Fig. 3B, the protein expression of PKA was increased by the 
A

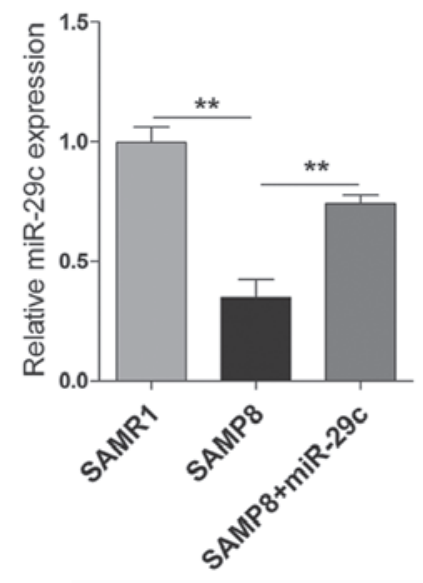

C

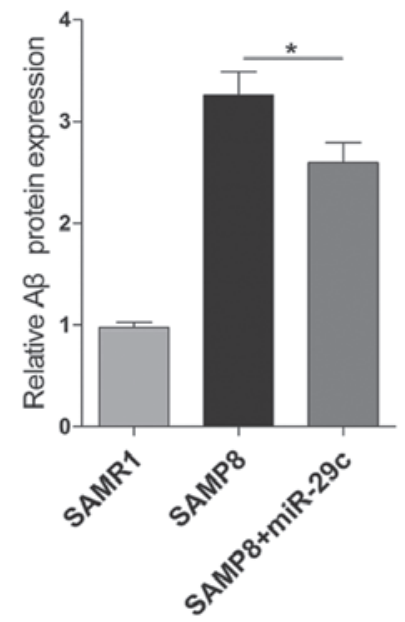

F

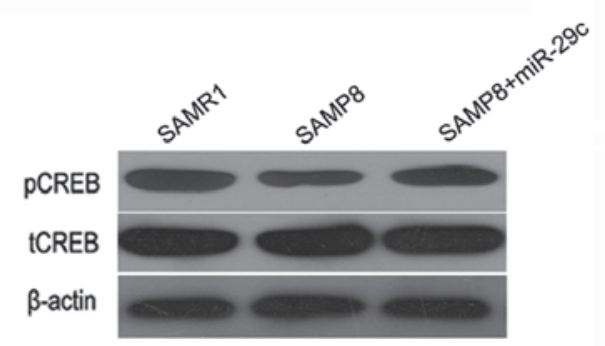

B

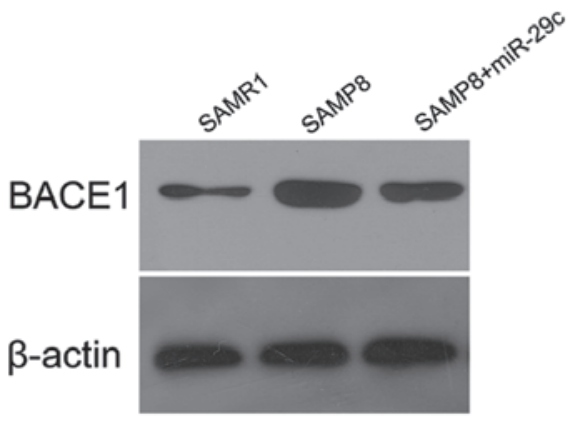

D
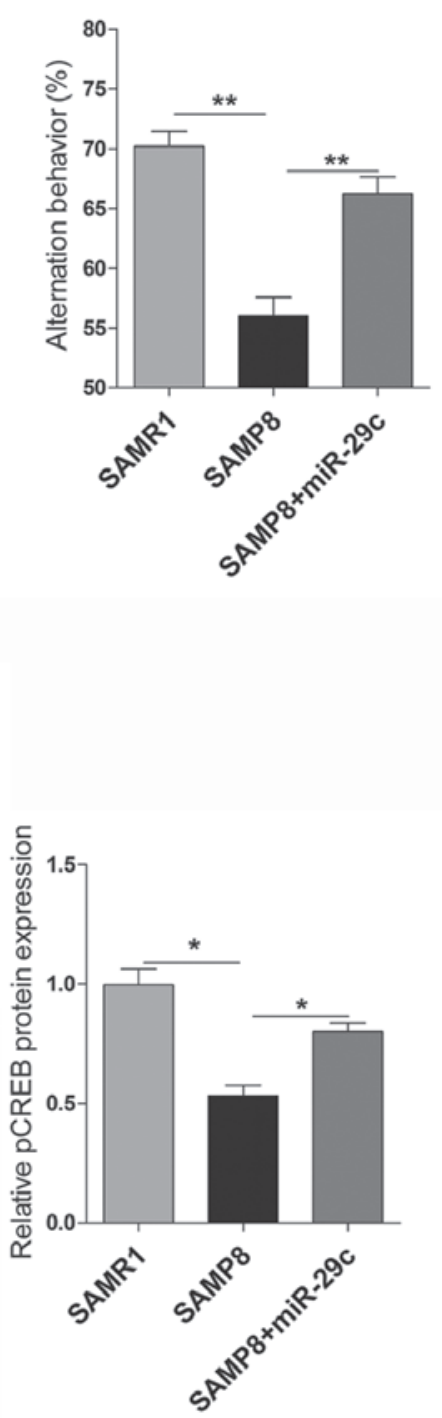

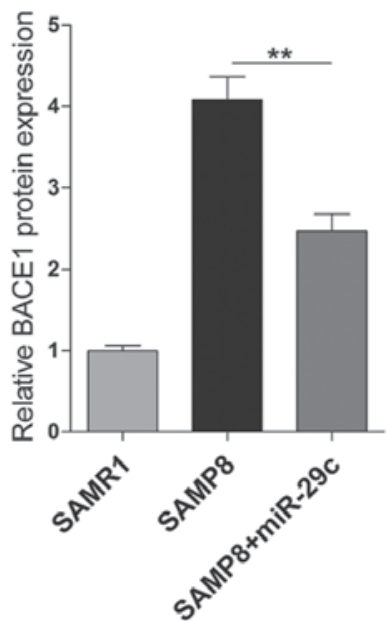

E
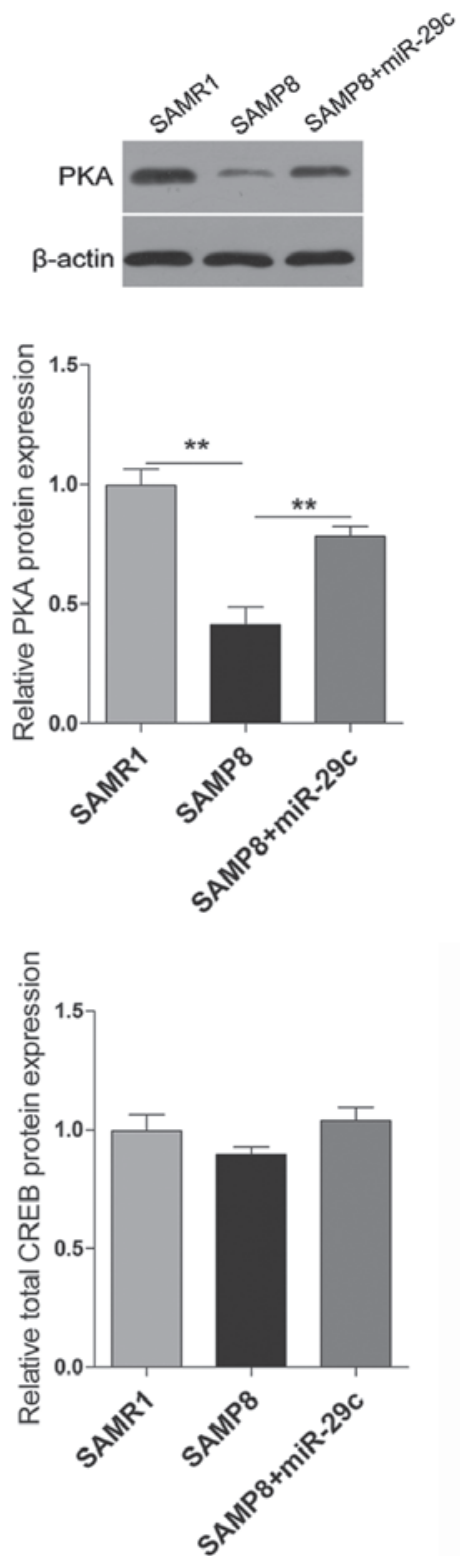

Figure 4. Effects of miR-29c in SAMP8 mice. (A) Expression levels of miR-29c in the hippocampi of SAMR1, SAMP8 and miR-29c-transfected-SAMP8 mice. (B) Protein expression levels of BACE1 in the hippocampi of SAMR1, SAMP8 and miR-29c-transfected-SAMP8 mice, and quantification of western blotting. (C) An ELISA was used to determine the protein expression of $\beta$-amylase in the hippocampi. (D) A Y-maze test was performed to measure the learning and memory behaviors of the mice. (E) Western blotting and quantification of the protein expression levels of PKA. (F) Western blotting and quantification of the protein expression levels of $\mathrm{pCREB}$ and tCREB. The data are expressed as the mean \pm standard deviation ( $\mathrm{P}<0.05$ and ${ }^{* * *} \mathrm{P}<0.01$, vs. control). PKA, protein kinase A; CREB, cAMP response element-binding protein; pCREB, phosphorylated CREB; tCREB, total CREB; miR, microRNA; BACE1, $\beta$-site amyloid precursor protein-cleaving enzyme 1. 
upregulation of miR-29c and decreased by the downregulation of miR-29c. Additionally, the expression of pCREB was induced significantly following transfection with pre-miR-29c, and was reduced following transfection with anti-miR-29c (Fig. 3C). These results suggested that miR-29c promoted hippocampal neuron cell proliferation by activating PKA signaling.

Effects of miR-29c on SAMP8 mice. The present study subsequently examined the effects of miR-29c in vivo, by injecting miR-29c mimics into the hippocampi of SAMP8 mice. The expression levels of miR-29c in the SAMP8 mice were significantly lower compared with those in the SAMR1 mice. When the miR-29c mimic was injected into the hippocampi of SAMP8 mice, the expression of miR-29c was significantly increased compared with the vehicle-injected control mice (Fig. 4A). Similar to the effects in vitro, the upregulation of miR-29c significantly decreased the protein expression levels of $\mathrm{BACE} 1$ and $\mathrm{A} \beta$ in vivo (Fig. $4 \mathrm{~B}$ and $\mathrm{C}$ ). The present study also examined the learning and memory behaviors of the SAMP8 mice 2 months following transfection with miR-29c. As shown in Fig. 4D, overexpression of miR-29c in the hippocampus promoted the learning and memory behaviors of the SAMP8 mice. Furthermore, PAK signaling was also examined, and the protein expression levels of PKA and pCREB were induced significantly by the upregulation of miR-29c (Fig. 4E and F).

\section{Discussion}

Current cerebrospinal fluid (CSF) and peripheral blood-based biomarkers, including $\mathrm{A} \beta, \mathrm{BACE} 1$ (13) and tau, can support the clinical diagnosis of AD with a sensitivity and specificity of $\sim 90 \%(14,15)$. Previous studies have focused on the involvement of miRNAs in AD. In a previous study, miRNAs with a differential expression in either the hippocampus or CSF from patients with AD and age-matched healthy control individuals were identified, the results of which suggested that low levels of miR-146a in the CSF were associated with AD (16). In the present study, miR-29c was significantly decreased in the peripheral blood of patients with AD compared with the age-matched control individuals, and was negatively correlated with an increased expression of BACE1. It was previously reported, using a microarray, containing 328 miRNAs, that reduced expression of the miR29a/b cluster is inversely correlated with the protein expression of BACE1 in the anterior temporal cortex of $\mathrm{AD}$ (5). The present study revealed no differential expression of $\mathrm{miR}-29 \mathrm{a} / \mathrm{b}$, however, differential expression of miR-29c was observed. A reasonable explanation is that the expression levels of miRNAs differs in different tissue samples. A previous study also demonstrated that miR-29c is reduced in the peripheral blood of patients with AD (17).

Increased expression of BACE1 has been previously observed in the brain, CSF and peripheral blood of patients with $\mathrm{AD}(18,19)$, suggesting that increased expression of BACE1 is an important risk factor for AD. The present study also revealed, using an ELISA, that the expression of BACE1 was increased in the peripheral blood of patients with AD. In a previous study, miR-29c, which is highly expressed in APPswe/PS $\triangle E 9$ mice, can lower the protein expression of BACE1 in vitro and in transgenic miR-29c mice (20). In primary cultured hippocampal neurons obtained from SAMR1 mice, the present study demonstrated that miR-29c regulated the expression of BACE1 at the transcriptional level by directly targeting its 3 'UTR. Furthermore, the upregulation of miR-29c also reduced the protein expression of $\mathrm{A} \beta$ by regulating BACE1. The present study provided evidence to suggest that low expression levels of miR-29c may have contributed to the deposition of $\mathrm{A} \beta$ by regulating BACE1 in the hippocampalneurons, which the downregulated miR-29c exhibited in the peripheral blood of patients with AD. Therefore, combined with previous studies, miR-29c may be a peripheral biomarker for $\mathrm{AD}$.

Patients with AD are characterized by learning and memory deficit, which is promoted by neurogenesis (21). The hippocampus is an important region of neurogenesis in adulthood (22). Therefore, promoting the survival and proliferation of hippocampal neurons is critical for the treatment of AD. The present study demonstrated that upregulation of miR-29c increased the proliferation of primary cultured hippocampal neurons in vitro, whereas downregulation of miR-29c impaired cell growth. PKA/CREB signaling has been implicated in neuroprotection (23). It has been reported that induction of pCREB via PKA is important in N-methyl-D-aspartate receptor-mediated neuroprotection (24). Caffeine intake has been associated with a lower incidence of AD in humans, and it has been revealed that caffeine shifts the balance between neurodegeneration and neuronal survival towards the stimulation of pro-survival cascades and the inhibition of pro-apoptotic pathways in the striatum by increasing PKA and pCREB (25). In addition, several lines of evidence indicate that PKA/CREB are involved in $\mathrm{A} \beta$-trigged disruption of synaptic plasticity in AD (26), and the overexpression of BACE1 reduces the activity of PKA and pCREB, contributing to the memory and cognitive deficits typical of AD (27). Therefore, activation of the PKA/CREB pathway is beneficial against AD. The present study demonstrated that upregulation of miR-29c induced the expression levels of PKA and CREB in primary cultured hippocampal neurons in vitro, and this molecular mechanism was subsequently confirmed in vivo. Notably, significant reductions in BACE1 and $A \beta$ were demonstrated in the hippocampi of SAMP8 mice following the overexpression of miR-29c for 2 months. Furthermore, treatment with miR-29c resulted in a significant increase in expression levels of PKA and pCREB in the hippocampi of the SAMP8 mice. The upregulation of miR-29c also improved the learning and memory outcomes, which were determined using a Y-maze test, in the SAMP8 mice.

In conclusion, the results of the present study suggested that miR-29c, as a peripheral AD biomarker, promoted learning and memory behaviors in SAMP8 mice, which was associated with a decrease in the production of $\mathrm{A} \beta$ by targeting BACE1 and increasing the activity of PKA/CREB involved in neuroprotection. Therefore, miR-29c may be a promising potential therapeutic target against AD.

\section{References}

1. Maciotta S, Meregalli M and Torrente Y: The involvement of microRNAs in neurodegenerative diseases. Front Cell Neurosci 7: 265, 2013. 
2. Rao AT, Degnan AJ and Levy LM: Genetics of Alzheimer disease. AJNR Am J Neuroradiol 35: 457-458, 2014

3. Davinelli S, Calabrese V, Zella D and Scapagnini G: Epigenetic nutraceutical diets in Alzheimer's disease. J Nutr Health Aging 18: 800-805, 2014.

4. Wirth M, Villeneuve S, La Joie R, Marks SM and Jagust WJ: Gene-environment interactions: Lifetime cognitive activity, APOE genotype, and $\beta$-amyloid burden. J Neurosci 34: 8612-8617, 2014.

5. Hébert SS, Horré K, Nicolaï L, et al: Loss of microRNA cluster miR-29a/b-1 in sporadic Alzheimer's disease correlates with increased BACE1/beta-secretase expression. Proc Natl Acad Sci USA 105: 6415-6420, 2008.

6. Selbach M, Schwanhäusser B, Thierfelder N, et al: Widespread changes in protein synthesis induced by microRNAs. Nature 455 58-63, 2008

7. Fineberg SK, Kosik KS and Davidson BL: MicroRNAs potentiate neural development. Neuron 64: 303-309, 2009.

8. Hampel H, Shen Y, Walsh DM, et al: Biological markers of amyloid beta-related mechanisms in Alzheimer's disease. Exp Neurol 223: 334-346, 2010

9. Wang WX, Rajeev BW, Stromberg AJ, et al: The expression of microRNA miR-107 decreases early in Alzheimer's disease and may accelerate disease progression through regulation of beta-site amyloid precursor protein-cleaving enzyme 1 . J Neurosci 28: 1213-1223, 2008.

10. Kocerha J, Kauppinen S and Wahlestedt C: microRNAs in CNS disorders. Neuromolecular Med 11: 162-172, 2009.

11. National Research Council (US) Committee for the Update of the Guide for the Care and Use of Laboratory Animals: Guide for the Care and Use of Laboratory Animals. 8th edition, National Academies Press, Washington DC, US, 2011.

12. Livak KF and Schmittgen TD: Analysis of relative gene expression data using real-time quantitative PCR and the 2(-Delta Delta C(T)) Method. Methods 25: 402-408, 2001.

13. Decourt B, Walker A, Gonzales A, et al: Can platelet BACE1 levels be used as a biomarker for Alzheimer's disease? Proof-of-concept study. Platelets 24: 235-238, 2013.

14. Shaw LM, Vanderstichele H, Knapik-Czajka M, et al: Alzheimer's Disease Neuroimaging Initiative: Cerebrospinal fluid biomarker signature in Alzheimer's disease neuroimaging initiative subjects. Ann Neurol 65: 403-413, 2009

15. Bibl M, Esselmann H, Lewczuk P, et al: Combined Analysis of CSF Tau, $\mathrm{A} \beta 42, \mathrm{~A} \beta 1-42 \%$ and $\mathrm{A} \beta 1-40 \%$ in Alzheimer's Disease, Dementia with Lewy Bodies and Parkinson's Disease Dementia. Int J Alzheimers Dis pii: 761571, 2010.
16. Müller M, Kuiperij HB, Claassen JA, Küsters B and Verbeek MM: MicroRNAs in Alzheimer's disease: Differential expression in hippocampus and cell-free cerebrospinal fluid. Neurobiol Aging 35: 152-158, 2014.

17. Bettens K, Brouwers N, Engelborghs S, et al: APP and BACE1 miRNA genetic variability has no major role in risk for Alzheimer disease. Hum Mutat 30: 1207-1213, 2009.

18. Marques SC, Lemos R, Ferreiro E, et al: Epigenetic regulation of BACE1 in Alzheimer's disease patients and in transgenic mice. Neuroscience 220: 256-266, 2012

19. Cattabeni F, Colciaghi F and Di Luca M: Platelets provide human tissue to unravel pathogenic mechanisms of Alzheimer disease. Prog Neuropsychopharmacol Biol Psychiatry 28: 763-770, 2004.

20. Zong Y, Wang H, Dong W, et al: miR-29c regulates BACE1 protein expression. Brain Res 1395: 108-115, 2011.

21. Cogswell JP, Ward J, Taylor IA, et al: Identification of miRNA changes in Alzheimer's disease brain and CSF yields putative biomarkers and insights into disease pathways. J Alzheimers Dis 14: 27-41, 2008.

22. Moon M, Cha MY and Mook-Jung I: Impaired hippocampal neurogenesis and its enhancement with ghrelin in 5XFAD mice. J Alzheimers Dis 41: 233-241, 2014.

23. Shi YQ, Huang TW, Chen LM, et al: Ginsenoside Rg1 attenuates amyloid-beta content, regulates PKA/CREB activity and improves cognitive performance in SAMP8 mice. J Alzheimers Dis 19: 977-989, 2010.

24. Valera E, Sánchez-Martín FJ, Ferrer-Montiel AV, Messeguer A and Merino JM: NMDA-induced neuroprotection in hippocampal neurons is mediated through the protein kinase $A$ and CREB (cAMP-response element-binding protein) pathway. Neurochem Int 53: 148-154, 2008.

25. Zeitlin R, Patel S, Burgess S, Arendash GW and Echeverria V: Caffeine induces beneficial changes in PKA signaling and JNK and ERK activities in the striatum and cortex of Alzheimer's transgenic mice. Brain Res 1417: 127-136, 2011.

26. Sierksma AS, Rutten K, Sydlik S, et al: Chronic phosphodiesterase type 2 inhibition improves memory in the APPswe/PS1dE9 mouse model of Alzheimer's disease. Neuropharmacology 64: 124-136, 2013.

27. Chen Y, Huang X, Zhang YW, et al: Alzheimer's $\beta$-secretase (BACE1) regulates the CAMP/PKA/CREB pathway independently of $\beta$-amyloid. J Neurosci 32: 11390-11395, 2012. 